PROFESIONALES Y HERRAMIENTAS PARA EL DESARROLLO LOCAL Y SUS SINERGIAS TERRITORIALES. EVALUACIÓN Y PROPUESTAS DE FUTURO IX Coloquio Nacional de Desarrollo Local del GTDL-AGE 

ANTONIO MARTÍNEZ PUCHE, XAVIER AMAT MONTESINOS, ISABEL SANCHO CARBONELL y DANIEL SANCHIZ CASTAÑO (EDS.)

\section{PROFESIONALES Y HERRAMIENTAS PARA EL DESARROLLO LOCAL Y SUS SINERGIAS TERRITORIALES. EVALUACIÓN Y PROPUESTAS DE FUTURO}

IX Coloquio Nacional de Desarrollo Local del GTDL-AGE

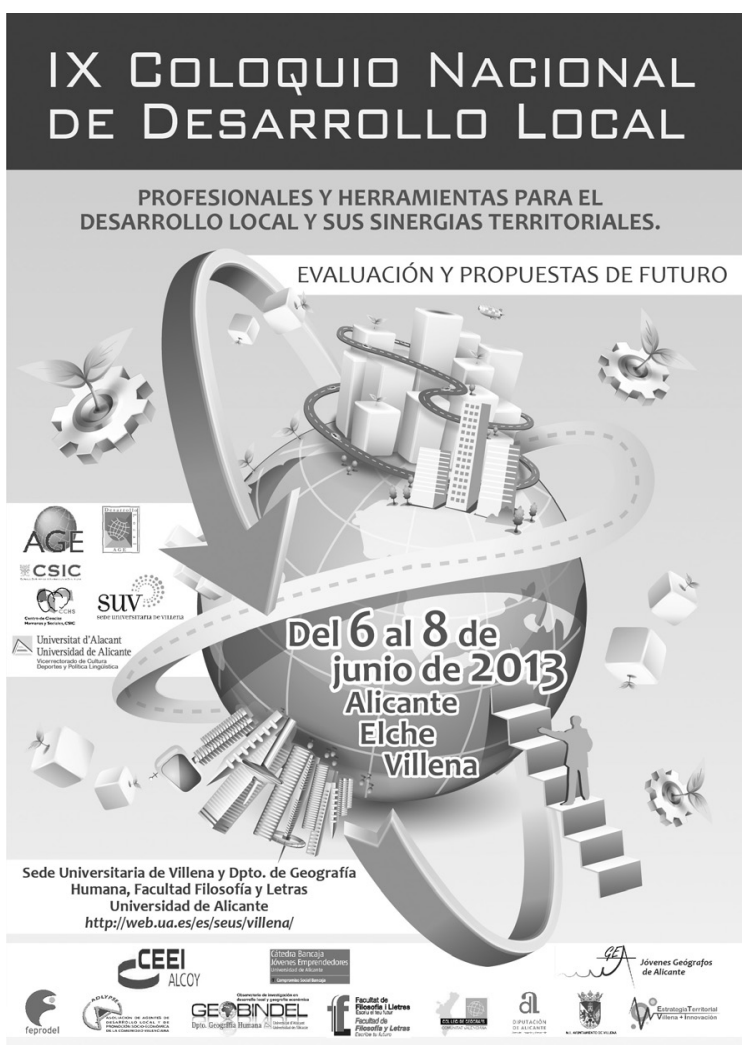


Este libro ha sido examinado y valorado por evaluadores ajenos a la Universidad de Alicante, con el fin de garantizar la calidad científica del mismo.

Publicacions de la Universitat d'Alacant

03690 Sant Vicent del Raspeig

Publicaciones@ua.es

http://publicaciones.ua.es

Telèfon: 965903480

(C) Antonio Martínez Puche, Xavier Amat Montesinos,

Isabel Sancho Carbonell y Daniel Sanchiz Castaño (eds.), 2016

(C) d'aquesta edició: Universitat d'Alacant

ISBN: 978-84-16724-00-0

Dipòsit legal: A 92-2016

Disseny de coberta: candela ink

Composició: Página Maestra (Miguel Ángel Sánchez Hernández)

Impressió i enquadernació: Guada Impresores

\section{unte \\ Unión de Editoriales
Universitarias Españolas \\ WWW.une.es
WWA}

Esta editorial es miembro de la UNE, cosa que garantiza la difusión y comercialización nacional y internacional de sus publicaciones.

Reservados todos los derechos. Cualquier forma de reproducción, distribución, comunicación pública o transformación de esta obra sólo puede ser realizada con la autorización de sus titulares, salvo excepción prevista por la ley. Diríjase a CEDRO (Centro Español de Derechos Repográficos, www.cedro.org) si necesita fotocopias o escanear algún fragmento de esta obra. 


\title{
LOS MANIFIESTOS PARA LA PROTECCIÓN AMBIENTAL EN CATALUNYA. BUSCANDO APROXIMACIONES A LA HIPÓTESIS DE LA RESILIENCIA
}

\author{
$M^{a}$ Àngels Alió Torres \\ Departament de Geografia Humana. Universitat de Barcelona \\ alio@ub.edu
}

\section{RESUMEN}

Las movilizaciones ciudadanas para la defensa del medio ambiente constituyen un fenómeno clave del proceso de transformación de la cultura y la mentalidad dominante, y, en particular, de la cultura ambiental y territorial. Esta comunicación se basa en un estudio del grupo de investigación sobre Ecología Social y Planeamiento Participativo de la Universitat de Barcelona sobre los manifiestos que fueron redactados en el contexto de las movilizaciones de la sociedad catalana para la protección ambiental desde 1972 hasta la actualidad.

Uno de los resultados preliminares de esta investigación se refiere a los contenidos de estos textos. De los cuales queremos destacar el carácter dual de muchos de ellos qué, además de la denuncia de las intervenciones y proyectos de gran impacto ambiental y territorial que actuaron como detonantes de la movilización, también contienen proposiciones y alternativas de actuación asociadas a las relaciones entre la sociedad y la naturaleza.

Palabras clave: Ecología Social, Participación Ciudadana, impacto ambiental, herramientas.

\section{MANIFESTS ENVIRONMENTAL PROTECTION IN CATALUNYA. LOOKING APPROACHES TO THE ASSUMPTIONS OF RESILIENCE}

\section{Abstract}

The civic mobilizations for the defense of the environment constitute a key phenomenon of the process of transformation of the culture and the dominant 
mentality, and, in particular, of the environmental and territorial culture. This is based on a study of the investigation group it has more than enough Social Ecology and Participatory Planning of Universitat of Barcelona on the manifestos that were edited in the context of the mobilizations of the Catalan society for the environmental protection from 1972 until the present time.

The communication presents a study of the investigation group it has more than enough Social Ecology and Participatory Planning of Universitat of Barcelona. This text talks about the environmental protection in Catalunya. This text speaks of the environmental contemporary culture that is together to the social (environmentalist, citizens and popular) movements and to the territorial and local sensibilities. tools.

Key words: Social ecology, Civic Participation, environmental impact,

1. Los MANifiestos PARA LA PROTECCión DEL MEDIO AMBIENTE DE CATALUNYA DESDE UNA PERSPECTIVA CRONOLÓGICA

Este conjunto de texto está formado por 138 manifiestos y declaraciones redactados por la sociedad civil catalana en situaciones de conflicto ambiental ${ }^{1}$. Cabe señalar que esta investigación forma parte de una investigación más amplia sobre las movilizaciones de reivindicación popular y ciudadana, y su papel como entornos favorables al desarrollo de las nuevas mentalidades y cultura ambiental (A. Alió y G. Jori, 2007 y 2008; A. Alió, 2011; G. Jori, 2011). En este contexto, estos documentos conforman una base documental muy valiosa en la que se encuentra escrita la percepción de las poblaciones locales sobre los problemas que podían generarse de la construcción de toda una serie de obras e infraestructuras que, desde mediados del siglo pasado hasta la actualidad, parecen estar conformando el modelo hegemónico de la ordenación económica territorial. Y también, lo que quizás es más interesante a efectos de esta ponencia, sobre otro desarrollo posible pero que las obras en cuestión estaban obstaculizando.

A modo de introducción a este conjunto de documentos es interesante detenerse en su desarrollo cronológico que sigue un estrecho paralelismo con las secuencias históricas básicas de los últimos cincuenta años (Figura 1).

1 En la fase inicial de este proyecto se contó con la inestimable colaboración de Sebastià Riutor, entonces becario de la Universitat de Barcelona para este proyecto. Actualmente, estos 138 documentos se han editado de forma telemàtica en el Centre de Recursos Telemàtic en Ecologia Social, disponible en www.ub.edu/cre 


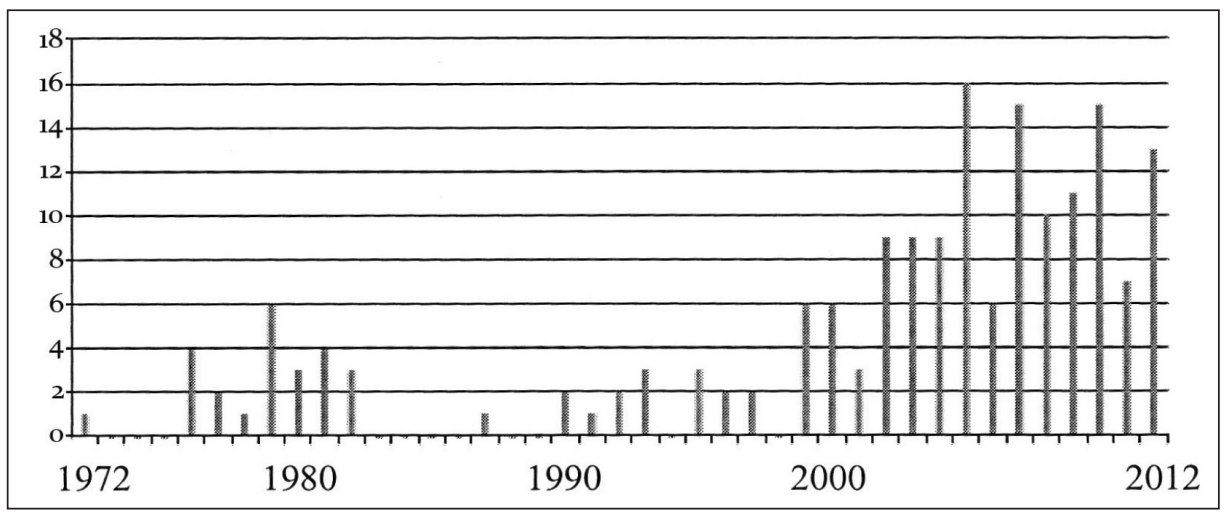

Figura 1. Cronología de los manifiestos para la protección del medio ambiente en Catalunya. $1972-2012^{2}$

Fuente: elaboración propia

Concretamente, pueden distinguirse tres grandes fases: La primera, coincidente con los últimos años del franquismo, que fueron también los de la implantación de los monopolios industriales que ya estaban empezando a articularse en la escala internacional. Al tiempo que también hacía eclosión en España el ecologismo contemporáneo, centrado muy especialmente en la protección de los ecosistemas naturales frente al avance de la urbanización especulativa y la industria energética nuclear ${ }^{3}$; La segunda, se desarrolla al compás del periodo de la transición que, de alguna manera, parecía haber facilitado una tregua entre las visiones contrapuestas de los dos modelos territoriales y socio-ambientales, repercutiendo, por tanto, en consensos, probablemente parciales, pero que habrían permitido abrir caminos hacia modelos de acción más armónicos con la naturaleza ${ }^{4}$ y; La tercera, la eclosión de un amplio movimiento ciudadano en constate crecimiento, transversal en todas las capas del tejido social, claramente implicado en la transformación hacia nuevas formas económicas más acordes con el modelo de la sostenibilidad. Respecto a esta última fase, hay que recor-

2 Este gráfico se basa en la versión actualizada de 175 documentos (ver nota anterior), 37 más que el conjunto de 138 que estamos tratando. Esta segunda cronología más completa sigue las tendencias generales de la cronología inicial y permite observar con más detalle el desarrollo histórico de este apartado.

3 Los primeros manifiestos ecologistas en el estado español fueron la Declaració d'AEORMA i Manifest de Benidorm (Declaración de AEORMA y Manifiesto de Benidorm) de 1974 y las Bases de Daimiel de 1978.

4 Entre otros datos, se podría recordar la primera moratoria nuclear en España, el compromiso internacional de la primera Cumbre de Río de Janeiro y, en 1997 la llegada a los acuerdos de la Cumbre de Kyoto, que luego el Estado Español no cumplió. También en Catalunya se generaron algunos acuerdos parciales, como el de los residuos a principios de los noventa, que también empezó a resquebrajarse pocos años después. 
dar algunos factores que explican esta eclosión de documentos. El primero, que las políticas públicas se anclaron en el modelo dominante de crecimiento y explotación de la naturaleza sin consideración alguna por la crisis ambiental, de graves implicaciones en la sociedad y alcance mundial (A. Vilches, 2009). En segundo lugar, el importante componente cultural, propio de los nuevos movimientos sociales, que refuerzan la reflexión y la preocupación por los valores inmateriales (R. Inglehart, 1987) y se concretan en temas tales como son la defensa del bien común, el decrecimiento, la austeridad y la justicia ambiental. Todas ellas asociadas a la nueva dialéctica de la historia, que enlaza la dimensión global con la preocupación y reivindicaciones asociadas a las relaciones locales entre sociedad y naturaleza y las reivindicaciones socioterritoriales. En el que es imprescindible contar con documentos breves y sencillos, generados por los mismos actores sociales que están sosteniendo las reivindicaciones e impulsan las acciones hacia un nuevo modelo de relación sociecoambiental 5 .

2. LOS MANIFIESTOS COMO DECOUMENTOS EN LA INTERFACE ENTRE LA DENUNCIA Y LAS FORMULACIONES PROPOSITIVAS

Desde el principio del análisis de los contenidos de este conjunto de texto hemos venido afirmado que los manifiestos pueden asimilarse a un intento por escribir nuevas ideas. Las cuales se asocian a objetivos y aspiraciones que los redactores de estos documentos conciben precisamente en base al deseo de transformar una realidad de la que los redactores se sienten insatisfechos (G. Jori, 2011). Y ello vale tanto para los manifiestos políticos, artísticos, culturales y científicos y, por supuesto, los manifiestos ambientalistas, considerando toda la amplia gama que va desde los proteccionistas y ecologistas a los de la justicia y la nueva cultura del territorio.

Vale la pena detenerse en esta breve consideración general de lo que significan los manifiestos y las declaraciones porqué nos permite destacar una característica que les es muy común. Se trata de su ambivalencia entre lo que se está denunciando y lo que se desearía alcanzar. O lo que es lo mismo, en la interface entre la oposición y las alternativas. Y que en el caso de los manifiestos que estamos estudiando, comporta una labor de redacción compleja por cuanto atañe al amplio marco de relaciones entre el mundo de lo social y de la naturaleza.

Ello nos aleja de la visión más común que entiende a los manifiestos para la protección del medio ambiente como simple oposición a proyectos o a grandes infraestructuras de las que tan sólo se tratan sus impactos ambientales. En este contexto, es necesario explicar que muchos de los manifiestos que hemos teni-

5 En muy pocos casos disponemos de los nombres de las personas que redactaron la versión finales de estos textos. Que siempre son firmados por las asociaciones o colectivos que promovieron los escritos. 
do la oportunidad de recopilar no son tan sencillos, en el sentido que tan sólo denuncien el proyecto o la actuación a la que se están oponiendo. Antes bien, muchos de ellos, también tienen un carácter marcadamente propositivo. Esto puede comprobarse por dos caminos metodológicos diferentes: el del análisis del discurso y el de la complejidad temática. Las características de esta comunicación hacen inviable la primera opción, pero sí que nos permiten avanzar este segundo camino más cuantitativo.

Así, conviene señalar que un primer resultado de nuestro análisis consistió, precisamente, en reconocer que existían dos grandes subgrupos de documentos. De una parte, todos aquellos a los que podría atribuirse fácilmente una temática que los definía propiamente. Eran, por ejemplo, los manifiestos especializados en los impactos de los proyectos de vías de circulación rápida, los que se centraban en los impactos de los trasvases y la gestión centralizada del agua, o los que reclamaban la protección de espacios naturales, o los antinucleares. Todos estos textos constituyen algo más de la mitad de la base de datos que estamos estudiando. Y queda todavía casi otra mitad, exactamente 64 textos que, además del tema central o dominante, por lo general recogido en el título, también prestan especial atención a otros temas. Ello nos llevó a desarrollar dos tipos de análisis diferentes pero complementarios: el análisis del tema dominante ${ }^{6}$ y el de las diferencias entre los manifiestos unitemáticos y multitemáticos, que vamos a presentar a continuación (Tabla 1).

Tabla 1. Simplicidad y complejidad en los manifiestos

\begin{tabular}{|l|c|c|}
\hline Manifiestos & Total & $\mathbf{\%}$ \\
\hline Unitemáticos & 74 & 53,6 \\
\hline Multitemáticos & $\mathbf{6 4}$ & $\mathbf{4 6 , 4}$ \\
\hline Dos temas & 30 & 21,7 \\
\hline Tres temas & 23 & 17,6 \\
\hline Complejos (+ 3 temas) & 11 & 7,1 \\
\hline Total & $\mathbf{1 3 8}$ & $\mathbf{1 0 0}$ \\
\hline
\end{tabular}

Fuente: elaboración propia.

En esta tabla se observa que los manifiestos multitemáticos permiten diferenciar hasta tres subgrupos. El más numeroso es el que está constituido por documentos que tratan dos temas. Uno de ellos, relativo a la actuación o proyecto que había provocado la oposición de la comunidad, y que fue el que abrió las puertas a la movilización. Correspondiendo lógicamente al segundo tema

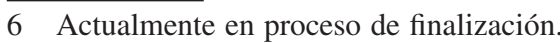


aquellos aspectos relacionados con las alternativas o contrapartidas. Este mismo planteamiento se reproduce en los manifiestos que desarrollan tres temas, lógicamente más complejos. Aunque este calificativo lo hemos reservado para los manifiestos con cuatro, cinco e incluso seis temas (Anexo 7) ${ }^{7}$, que empiezan a aparecer con el nuevo milenio. Entre éstos se puede distinguir dos subgrupos: Los que se refieren exclusivamente a demarcaciones comarcales (Empordà, Anoia, Penedès y Lleida); Y los que plantean específicamente el desarrollo de la nueva cultura del territorio en el ámbito catalán.

Antes de terminar este punto, solo quisiéramos poner la atención en los esfuerzos que, presumiblemente, se hubieron de dedicar a la elaboración de la mayoría de estos documentos, tanto en el sentido de la redacción, como en el de conseguir acuerdos y complicidades entre las poblaciones directamente implicadas. Con el objetivo que dichos textos hicieran posible la reflexión y proporcionasen coherencia racional a la movilización explicando las interrelaciones entre lo que estaban rechazando y lo deseaban. Entre las que destacan, por supuesto, las relaciones sociedad-medioambiente y sus formas de concreción en lugares y territorios. Lo que nos llevaría a la consideración de todos estos textos como una pieza literaria o documental clave del proceso de desarrollo de lo que llamamos nueva cultura del territorio.

\section{LA HIPÓTESIS DE LA RESILIENCIA}

Siguiendo las pistas de la nueva cultura del territorio, por cierto muy bien representada en los manifiestos que hemos denominado complejos, parecería que podemos preguntarnos por su posible función en el desarrollo de las resiliencias socioterritoriales. Lo que nos llevaría a pensar que un modelo de desarrollo local en armonía con sus ecosistemas naturales es un instrumento de la sociedad que surge y se desarrolla en un contexto de resistencias y resiliencias múltiples y en interacción. Las cuales, a su vez, se generan desde diversos sectores y con distintas características ya que, además de las resiliencias económicas, también hay las resiliencias culturales, políticas, artísticas y personales.

A nuestro pesar, debemos reconocer que no estamos preparados para responder con precisión. Y es que, de hecho, tampoco sabemos si el modelo de la resiliencia nos puede ayudar a estudiar mejor los procesos de cambio de nuestra sociedad hacia nuevas formas de relación socioambiental y territorial. Aunque, de hecho, existen trabajos científicos que están afirmando que podría tener esta capacidad.

En cualquier caso, nos hemos propuesto experimentar con las posibilidades metodológicas de este concepto intentado tender un puente entre nuestra inves-

7 Para la elaboración de los anexos hemos contado con la ayuda de Hozana Raquel de Medeiros García. 
tigación y el marco teórico disponible. En este punto podríamos asumir que la ciencia distingue entre tres tipos de resiliencia.

La primera, la resiliencia que proviene de las ciencias físicas, que tienen su origen en la lengua latina, y enfatiza el significado de volver atrás de un salto, ser repelido o surgir. A Forés (2008) explica que esta definición surge de la física, la mecánica y la metalúrgica, cuando quieren destacar la capacidad de los metales de resistir un impacto y recuperar su estructura original. Esta dialéctica entre la resistencia a los impactos y la capacidad de resurgir, o regenerarse, se destaca también como una de las definiciones esenciales de la resiliencia por parte de las ciencias sociales. Psicólogos y pedagogos se cuentan entre los primeros en investigar la capacidad humana para sustraerse a las desgracias, es decir para resistir, y desarrollar sus vidas en un proceso resiliente que, según B. Cyrulnik, permite unir, dentro de lo posible, las partes de la personalidad que fueron destrozadas por el trauma. La diferencia con las ciencias físicas vendría de la mano de la importancia de los fenómenos social y cultural. Stephan Vanistendaelm por ejemplo, señala la importancia de los procesos de creatividad y de desarrollo positivo, que acercan la resiliencia al arte y la transforman en acciones bellas y positivas. La cual, en suma, vendría a ser como los factores que ayudarían a unir lo que fuera roto o desestructurado. Si bien, al menos que tengamos conocimiento, siempre se han referido al ámbito de lo individual.

Se dispone, además de una tercera definición que parecería muy adecuada para plantear las resiliencias territoriales. Se trata de la definición que se está difundiendo en el marco del movimiento de la permacultura, que toma la idea de la resiliencia de la biología, pero que la reformula en el marco de las sociedades actuales para hacer frente a la crisis ambiental y energética. A destacar, dos rasgos esenciales que pueden ser de gran utilidad para los planteamientos de las estrategias territoriales. Uno, el hecho que plantee la resiliencia como capacidad de las comunicaciones humanas, y no sólo de los individuos. Y el otro, que al centrarse en situaciones de crisis ambiental, parece que esté proponiendo una idea de resiliencia que no se centra exclusivamente en el medio físico o en el social, sino que esté proponiendo la interrelación dialéctica entre los dos medios como la fuente misma de resiliencia. Podríamos preguntarnos si según este planteamiento estaríamos asistiendo a una versión contemporánea del modelo biologista decimonónico. O también que quizás estamos ante un planteamiento epistemológico cientificista que desarrolla los criterios ecologistas de los sesenta en el siglo pasado. Algo de todo ello hay probablemente. Pero también está fuera de duda que desde esta óptica la resiliencia está ganando terreno, en particular en los espacios urbanos, precisamente donde es más difícil hablar de procesos de readaptación a los ecosistemas naturales.

Rob Hopkins (A. Scotti, 2011) ha sido uno de los autores que más ha contribuido a la difusión de esta concepción de la resiliencia. Añadiendo matices muy interesantes al proponer que los lugares y la ciudad contemporánea en 
crisis pueden transformase en ciudades y lugares resilientes si cambian una serie de comportamientos y características. Es decir, que pueden transformarse en pueblo y ciudades que al tiempo que empiezan a adaptarse a las nuevas condiciones de decrecimiento y de crisis ambiental empiezan a resolver muchos de los problemas y rupturas a que deben enfrentarse los espacios locales y los territorios actuales. En la medida que esta definición está directamente relacionada con las acciones en la esfera local y la crisis ambiental, podría ser conveniente señalar que estos dos autores insisten en la necesidad que las sociedades locales sean quienes establezcan las pautas para la implementación de los cambios y los nuevos comportamientos.

\section{El PAPEl DE LA SOCIEDAD EN LSO MANIFIESTOS PARA LA PROTECCIÓN AMBIENTAL}

Por consiguiente, puede ser oportuno preguntarnos por el papel que este conjunto de manifiestos atribuyen a la sociedad en este proceso de generación y desarrollo de nuevas alternativas y posibilidad. Con este objetivo vamos a presentar unos resultados preliminares que se pudieron obtener después de una lectura pormenorizada de estos 138 primeros manifiestos con el objetivo de identificar las referencias sociales contenidas en los textos. Entendidas éstas en el sentido amplio de la palabra social.

Como primer resultado podemos decir que casi las dos terceras partes, concretamente 85 manifiestos, tratan de manera explícita factores y fenómenos sociales. Lo cual desmiente la idea muy extendida de los manifiestos ambientalistas como un conjunto de textos exclusivamente centrados en la defensa de los paisajes naturales y la biodiversidad.

Un segundo resultado muy interesante se centra en las diferencias temáticas y conceptuales de estos planteamientos sociales, que tienen una gran diversidad. Aun cuando, por lo general, tienda a dominar un discurso económico en la línea comprehensiva de la definición griega clásica de la palabra economía 8 . Ello se expresa en la tabla 2, que muestra en primer lugar el predominio numérico de un conjunto heterogéneo de conceptos e ideas de carácter económico. Las cuales constituyen un grupo característico que incluye reflexiones críticas sobre factores económicos como son el consumo irresponsable, el trabajo alienado, la distribución desigual de la riqueza, también dentro de un mismo municipio o comarca, la especulación y el crecimiento económico. Pero que también propone nuevas formas de intervenir en el sistema económico, como son el turismo rural, el transporte público', y las tasas ambientales.

8 Recordemos que la definición clásica griega de la palabra economía se centra en las relaciones de sustentabilidad entre las personas y el entorno, y que ha sido recuperada actualmente en el marco de la corriente de la economía ecológica.

9 Se han identificado hasta 20 referencias a la crítica del transporte privado y la necesidad de promover el transporte público. 
Tabla 2. Aproximación cuantitativa a la dimensión social de los manifiestos

\begin{tabular}{|l|c|}
\hline \multicolumn{1}{|c|}{ Tema } & $\mathbf{N}^{\mathbf{0}}$ de referencias \\
\hline Conceptos económicos en general & 61 \\
\hline Agricultura & 38 \\
\hline Patrimonio y bienes comunes & 29 \\
\hline Visión de futuro & 16 \\
\hline Ética y solidaridad & 14 \\
\hline
\end{tabular}

Fuente: elaboración propia.

Veríamos a continuación otro grupo que también guarda relación con la economía pero que se ha preferido tratar aparte. Se trata básicamente de 25 manifiestos centrados en temas agrarios que, a su vez, pueden diferenciarse según traten exclusivamente de las agriculturas comarcales o bien del conjunto de Catalunya (Anexo 2). Todos ellos, en conjunto, proporcionan hasta un total de 38 explicaciones alusivas a la agricultura que son, quizás, las que están más en la línea del modelo de desarrollo local y la concepción de la permacultura sobre la resiliencia. Ello se refleja en el tratamiento que hacen de las relaciones entre la agricultura y el medio ambiente, la población local y el campesinado.

También hay otros tipos de reflexiones que merecen nuestra atención. Entre éstos, merece especial atención una serie de manifiestos que hablan explícitamente de nuevos planteamientos territoriales, hasta 29 referencias, y que formulan ideas y conceptos relacionados con el patrimonio público y los bienes comunes. Normalmente, se trata de manifiestos que usan el concepto de patrimonio como sinónimo de paisaje, en la línea de la Directiva Europea, en la que confluyen los aspectos ecosistémicos e históricos, como son las arquitecturas autóctonas, los monumentos y las redes locales y comarcales de abastecimiento y distribución de agua. También se incluyen en esta categoría las referencias a los bienes comunes, como son el mar, el agua y el aire y, por supuesto, la calidad de vida, que siempre va asociada al buen estado de los ecosistemas, así como los que hablan de los valores sociales y culturales de las comunicaciones. Por descontado, la mayoría de manifiestos que tratan el tema de la agricultura, a los que acabamos de hacer mención, podrían también sumarse a este grupo.

Y para terminar, hay que hacer mención de dos subconjuntos más de referencias y consideraciones sociales. El de los manifiestos que tratan de las perspectivas de futuro, incluyendo conceptos como son la sostenibilidad y la responsabilidad respecto a las generaciones futuras, todos ellos en alusión a modelos territoriales y de desarrollo local que se proyectan sociales y cualitativas, en la línea de los valores y éticas, como son la solidaridad intermunicipal, 
la ética y los derechos ambientales, la distribución equitativa de las rentas y salarios y la denuncia de la especulación y el despilfarro de los recursos naturales.

\section{A MODO DE CONLUSIÓN}

Ya para terminar, sólo nos queda por destacar dos temas. El primero, la importancia de los manifiestos como documentos que recogen resistencias de los sectores de población en relación a los proyectos públicos y de los grandes grupos empresariales. Al tiempo que les llevan a formular propuestas y formulaciones activas sobre el tipo de actuaciones que desearían para sus territorios. Desde luego, se trata de un tema fundamental en geografía, en la medida que conecta los espacios vividos y el espacio de uso con el de la naturaleza y los recursos naturales. Y que, por tanto, permite adentrarnos en el conocimiento de los procesos que gravitan en torno a lo que denominamos nueva cultura del territorio.

Pero también he intentado tender un puente con la hipótesis de la resiliencia y lo hemos hecho bosquejando dos caminos, necesariamente provisionales. Uno, en de la misma definición del concepto, cuya aplicación en nuestro campo parece que debería situarse en el terreno de las condiciones sociales de la comunidad aun cuando existe todavía un campo muy grande sobre el que preguntarse e investigar. Y el otro, el de los mismos manifiestos como instrumento de resiliencia cultural en situaciones graves conflicto territorial. En este segundo sentido hemos intentado desbrozar esta hipótesis partiendo de la base que los mismo textos podían iluminar algunas ideas sobre los comportamientos, estrategias o posibilidad de resiliencia de nuestra sociedad en la era actual.

\section{BiBLIOGRAFÍA}

ALIÓ, A.: "La paradoxa ambiental urbana: aproximaciones a la formació de les noves cultures ambientals des de la crisi dels residus". En: A. Alió i G. Jori (Eds.): Les societats urbanes davant la reforma ambiental: visions $i$ propostes al voltant de la sostenibilidad. Barcelona, Universitat de Bancelona, 2011. pp. 138-175. (disponible en Internet: http://www.ub.edu/cres/)

ALIÓ, A. - JORI G.: "Hacia una hipótesis del modelo de transición ambiental: El protagonismo de las movilizaciones ecologistas". XX Congreso de Geógrafos Españoles. Sevilla, 2007.

ALIÓ, A. - JORI, G: "Evolución de las movilizaciones ambientales en Catalunya entre 1970 y 2007”. En: JM. Feria Toribio, A. García y J. Ojeda (Eds.): Territorios, Sociedades y Políticas. Sevilla, Universidad Pablo de Olavide y Asociación de Geógrafos Españoles, 2009. pp. 431-441.

FORÉS, A.: "Pedagogía de la resiliencia". Revista Misión Joven. Barcelona, Universitat Ramon Llul. 377-2008. 
INGLEHART, R.: El cambio cultural en las sociedades industriales avanzadas. Madrid, Centro de Investigaciones Sociológicas - Siglo XXI, 1991, $529 \mathrm{pp}$.

JORI, G. "Manifiestos ecologistas y cultura ambiental. A propósito de un repertorio digital de manifiestos", Ar@cne. Revista Electrónica de Recursos en Internet sobre Geografía y Ciencias Sociales. Barcelona: Universidad de Barcelona, $\mathrm{n}^{\circ}$ 142, 1 de enero de 2011. <http://www.ub.es/geocrit/aracne/ aracne-142.htm>.

SCOTTI, A.: "Ciudades resilientes". En: A. Alió y G. Jordi (Eds.): Les societats urbanes davant la reforma ambiental: visions y propostes al voltat de la sostenibilitat. Barcelona, Universitat de Barcelona - Grup de Geògrafs per a l'Ecologia Social. Universitat de Barcelona, 2001. pp. 111-128. (disponible en Internet en: www.ub.edu/cres)

VILCHES, A. - MACÍAS, O y GIL PÉREZ, D.: Dëcada de la acción para la sostenibilidad. Madrid, Organización de Estados Iberoaméricanos, 2009. 141 pp. (disponible en Internet en: http://www.oei.es/DOCUMENTO1caeu.pdf). 


\section{ANEXOS}

Anexo 1. Manifiestos Complejos

\begin{tabular}{|c|c|}
\hline & Título de manifiesto \\
\hline 2001 & $\begin{array}{l}\text { Manifest. Aturem la destrucció del medi ambient - Contra la política } \\
\text { ambiental de la Generalitat } \\
\text { Manifiesto. Detengamos la destrucción del medio ambiente - Contra } \\
\text { la política ambiental de la Generalitat }\end{array}$ \\
\hline 2002 & $\begin{array}{l}\text { Manifest Salvem L'Empordà } \\
\text { Manifiesto. Salvemos L'Espordà }\end{array}$ \\
\hline 2003 & $\begin{array}{l}\text { Declaració de Figures per una nova cultura del territori } \\
\text { Declaración de Figueres por una nueva cultura del territorio }\end{array}$ \\
\hline 2003 & $\begin{array}{l}\text { Prou agressions al territori } \\
\text { Basta de agresiones al territorio }\end{array}$ \\
\hline 2005 & $\begin{array}{l}\text { Declaració per un desenvolupament sostenible de la comarca (Anoia) } \\
\text { Declaración por un desarrollo sostenible de la comarca (Anoia) }\end{array}$ \\
\hline 2005 & $\begin{array}{l}\text { Declaració de Tortosa per una nova cultura del territori } \\
\text { Declaración de Tortosa por una nueva cultura del territorio }\end{array}$ \\
\hline 2006 & $\begin{array}{l}\text { Manifest fundacional del Centre per la Sostenibilitat Territorial } \\
\text { Manifiesto fundacional del Centro para la Sostenibilidad Territorial }\end{array}$ \\
\hline 2006 & $\begin{array}{l}\text { Manifest per una nova cultura del territori } \\
\text { Manifiesto para una nueva cultura del territorio }\end{array}$ \\
\hline 2007 & $\begin{array}{l}\text { Manifest en defensa de les Terres de Ponent } \\
\text { Manifiesto en degensa de las Terres de Ponent }\end{array}$ \\
\hline 2008 & $\begin{array}{l}\text { Manifest de la Unió de Plataformes en defensa del medi natural, el } \\
\text { patrimoni cultural i els drets cívics (Manifest de Montserrat). Mani- } \\
\text { fiesto de la Unión de Plataformas en defensa del medio natural, el } \\
\text { patrimonio cultural y los derechos cívicos (Manifiesto de Montse- } \\
\text { rrat) }\end{array}$ \\
\hline 2009 & $\begin{array}{l}\text { Manifest contra la política ambiental del Govern de la Generalitat } \\
\text { Manifiesto contra la política ambiental del Gobierno de la Genera- } \\
\text { lidad }\end{array}$ \\
\hline
\end{tabular}

Fuente: elaboración propia. 
Anexo 2. Manifiestos que hacen un tratamiento de la agricultura

\begin{tabular}{|c|c|}
\hline & Título de manifiesto \\
\hline 1976 & Manifiesto sobre las zonas húmedas de la Costa Brava \\
\hline 1978 & $\begin{array}{l}\text { El futur de Gallecs només pot decidir-lo el poble } \\
\text { El futuro de Gallecs sólo puede decidirlo el pueblo }\end{array}$ \\
\hline 1979 & Comunicado de los grupos ecologistas catalanes a la opinión pública \\
\hline 1979 & $\begin{array}{l}\text { L'atur i les centrals nuclears } \\
\text { El paro y las centrales nucleares }\end{array}$ \\
\hline 1979 & $\begin{array}{l}\text { Manifest sobre l'abocador de Garraf } \\
\text { Manifiesto sobre el vertedero de Garraf }\end{array}$ \\
\hline 1980 & $\begin{array}{l}\text { Manifest final de la marza antinuclear de Catalunya } \\
\text { Manifiesto final de la marcha antinuclear de Catalunya }\end{array}$ \\
\hline 1982 & $\begin{array}{l}\text { Per la supervivencia de les Terres de l'Ebre. Manifest contra el fun- } \\
\text { cionament de la nuclear d'Acó } \\
\text { Por la supervivencia de las Terres del Ebre. Manifiesto contra el } \\
\text { funcionamiento de la nuclear de Ascó }\end{array}$ \\
\hline 1993 & $\begin{array}{l}\text { Defensem la nostra terra de la contaminació } \\
\text { Defendamos nuestra tierra de la contaminación }\end{array}$ \\
\hline 1999 & $\begin{array}{l}\text { Manifest del Priorat sobre les centrals eòliques } \\
\text { Manifiesto del Priorat sobre las centrales eólicas }\end{array}$ \\
\hline 2000 & $\begin{array}{l}\text { Manifest Comarcal en Favor de la Constitució d'un Consorci per } \\
\text { a la Protecció de l'Espai Natural (agrícola-forestal) del Montbaig- } \\
\text { Montpedrós-Puig Vicenç } \\
\text { Manifiesto Comarcal en Favor de la Constitución de un Consorcio } \\
\text { para la Protección del Espacio Natural (agrícola-forestal) del Mont- } \\
\text { baig-Montpedrós-^Puig Vicenç }\end{array}$ \\
\hline 2000 & $\begin{array}{l}\text { Manifest reivindicatiu de la Plataforma Salvem La Tordera } \\
\text { Manifiesto reivindicativo d ela Paltaforma Salvem La Tordera }\end{array}$ \\
\hline 2003 & $\begin{array}{l}\text { Declaració de L'Agulla } \\
\text { Declaración de La Agulla }\end{array}$ \\
\hline 2003 & $\begin{array}{l}\text { Crida en defensa del Vallès } \\
\text { Llamamiento en defensa del Vallès }\end{array}$ \\
\hline 2004 & $\begin{array}{l}\text { Manifest de Valbona de les Monges: Per un canal Segarra-Garrigues } \\
\text { pel Segle XXI } \\
\text { Manifiesto de Vallbona de les Monges: A favor de un canal Segarra- } \\
\text { Garrigues para el Siglo XXI }\end{array}$ \\
\hline
\end{tabular}




\begin{tabular}{|c|l|}
\hline 2004 & $\begin{array}{l}\text { Declaració de la lluita contra el túnel de Bracons } \\
\text { Declaración de la lucha contra el túnel de Bracons }\end{array}$ \\
\hline 2005 & $\begin{array}{l}\text { Manifest "Protegim L'Horta de Lleida" } \\
\text { Protejamos la Huerta de Lleida }\end{array}$ \\
\hline 2005 & $\begin{array}{l}\text { Manifest per la conservació de l'ós bru a Catalunya } \\
\text { A favor de la conservación del oso pardo en Cataluña }\end{array}$ \\
\hline 2007 & $\begin{array}{l}\text { Manifest del Vallès sense el Quart Cinturó } \\
\text { Manifiesto del Vallès sin el Cuarto Cinturón }\end{array}$ \\
\hline 2007 & $\begin{array}{l}\text { Manifest en defensa de les Terres de Ponent } \\
\text { Manifiesto en defensa de las Tierras de Ponent }\end{array}$ \\
\hline 2008 & $\begin{array}{l}\text { Manifest "Els transvasaments no són la solució" } \\
\text { Manifiesto "Los trasvases no son la solución” }\end{array}$ \\
\hline 2009 & $\begin{array}{l}\text { Manifest de la Plataforma per la Defensa de Gallecs } \\
\text { Manifiesto de la Plataforma para la defensa de Gallecs }\end{array}$ \\
\hline 2009 & $\begin{array}{l}\text { Manifest per l'Horta, por Lleida } \\
\text { Manifiesto por la Huerta, por Lleida }\end{array}$ \\
\hline 2009 & $\begin{array}{l}\text { Manifest de Bellpuig. Salut, emocràcia i Bons Aliments. } \\
\text { Manifiesto de Bellpuig. Salud, Democracia y Buenos Alimentos }\end{array}$ \\
\hline 2010 & $\begin{array}{l}\text { Manifest de la 1'a Assamblea de la Plataforma en Defensa del Ebre } \\
\text { Manifiesto de la 10a Asamblea de la Plataforma para la Defensa del } \\
\text { Ebro }\end{array}$ \\
\hline 2010 & $\begin{array}{l}\text { Rius amb aigua: Rius Vius } \\
\text { Ríos con agua: Ríos Vivos }\end{array}$ \\
\hline
\end{tabular}

Fuente: elaboración propia. 